\title{
Editorial
}

\section{Die Forschungsgruppe Search Studies an der HAW Hamburg}

https://doi.org/10.1515/iwp-2019-0003

In diesem Themenheft stellen wir aktuelle Beispiele der informationswissenschaftlichen Forschung aus der Forschungsgruppe Search Studies am Department Information an der Hochschule für Angewandte Wissenschaften Hamburg vor. Als Ergebnis können wir nun fünf Aufsätze präsentieren, die unterschiedliche Aspekte der angewandten informationswissenschaftlichen Forschung behandeln.

Die Forschungsgruppe Search Studies beschäftigt sich mit der Informationssuche vor allem in kommerziellen Suchmaschinen, aber auch in spezialisierten Suchsystemen. Aspekte der informationswissenschaftlichen Forschung rund um das Thema Suche umfassen nicht nur systemseitige, sondern auch gesellschaftliche Fragestellungen der Websuche sowie das Verhalten der Nutzerinnen und Nutzer bei ihrer Interaktion mit dem Suchsystem. Die Forschungsgruppe Search Studies bündelt die (bisher bereits am Department Information in der Forschung und Lehre behandelten) Bereiche (Interactive) Information Retrieval, Information Behaviour, Usability, Wissensorganisation und -repräsentation und führt sie in gemeinsamen Forschungs- und Kooperationsprojekten zusammen.

Eine Besonderheit der Forschung an einer Hochschule für Angewandte Wissenschaften ist der Transfer von Forschungsergebnissen in die Praxis und in die Lehre in Form von Forschungs- und Entwicklungsprojekten. Mit unterschiedlichen Partnern aus der Praxis haben sich zahlreiche, teilweise über Jahre andauernde Kooperationen ergeben, u. a. mit der Deutschen Telekom, der Otto GmbH \& Co. KG und der ZBW-Leibniz-Informationszentrum Wirtschaft. Von diesen Kooperationen profitieren neben den Projektpartnern und den Forschenden auch die Studierenden, die beispielsweise in den in den Master „Information, Medien,

*Kontaktperson: Prof. Dr. Dirk Lewandowski, Hochschule für Angewandte Wissenschaften Hamburg, Fakultät DMI, Department Information, Hamburg, E-Mail: dirk.lewandowski@haw-hamburg.de Prof. Dr. Ulrike Spree, Hochschule für Angewandte Wissenschaften Hamburg, Fakultät DMI, Department Information, Hamburg, E-Mail: ulrike.spree@haw-hamburg.de
Bibliothek“ integrierten Auftragsforschungsprojekten bei diesen Praxispartnern eigene hochschulgelenkte Forschungsprojekte durchführen können.

Drei der in diesem Themenheft enthaltenen Beiträge präsentieren Forschungsergebnisse, die im Rahmen von extern geförderten Drittmittelprojekten entstanden sind. Die Fördermittelgeber sind hier die Deutsche Forschungsgemeinschaft, das Bundesministerium für Ernährung und Landwirtschaft sowie die Dieter Schwarz Stiftung.

Die Hochschule für Angewandte Wissenschaften Hamburg bietet im Rahmen eines internen, kompetitiven Auswahlverfahrens die Möglichkeit, Stellen für Promovierende einzurichten. Diese halben Stellen werden für jeweils drei Jahre vergeben und ermöglichen die Promotion in Zusammenarbeit mit einer Universität. Zwei der Promovierenden, Friederike Hanisch und Sebastian Sünkler, sind an unterschiedlichen Beiträgen in diesem Themenheft als Mitautoren beteiligt; Christiane Behnert stellt ihr Promotionsprojekt in einem eigenen Beitrag vor.

\section{Beiträge in diesem Heft}

In dem Artikel „Anzeigenkennzeichnung auf Suchergebnisseiten: Empirische Ergebnisse und Implikationen für die Forschung“ berichten Dirk Lewandowski, Sebastian Sünkler und Friederike Hanisch über eine Studie, in der sie untersucht haben, inwieweit Suchmaschinennutzende die Erlösmodelle der Suchmaschinenbetreiber verstehen und ob sie in der Lage sind, Werbung auf den Suchergebnisseiten von den eigentlichen Suchresultaten zu unterscheiden. Hier zeigt sich, dass es erhebliche Schwierigkeiten gibt, werbefinanzierte Suchsysteme zu verstehen und damit kompetent zu nutzen. Daraus ergeben sich Konsequenzen für den derzeitigen Stand der Informationskompetenz und die Frage, wie diese gefördert werden kann. Folgen hat das aber auch für die theoretischen Modelle des Information Seeking, die bislang von Suchsystemen ausgehen, die ihren Nutzerinnen und Nutzern neutral gegenüberstehen und deren Betreiber bei der Darstellung der Ergebnisse keine Eigeninteressen verfolgen. 
Der Beitrag „Evaluierung von Rankingverfahren für bibliothekarische Informationssysteme“ von Kim Plassmeier, Christiane Behnert, Timo Borst und Dirk Lewandowski stellt Ergebnisse des DFG-geförderten Projekts LibRank vor, in dem Rankingverfahren für bibliothekarische Informationssysteme erprobt und evaluiert wurden. Neben dem Aufbau der Evaluierungen werden die Resultate der Evaluierungsläufe zusammengefasst und gezeigt, welchen Einfluss verschiedene Rankingansätze auf die Qualität der Suchergebnisse haben.

Unter dem Titel „Relevance Clues - Kriterien und Einflussfaktoren bei der Relevanzbewertung von Surrogaten in akademischen Informationssystemen“ stellt Christiane Behnert einen zentralen Aspekt ihres Promotionsprojektes vor. Sie greift ein grundlegendes Thema der Informationswissenschaft auf, nämlich die Bewertung der Relevanz von Dokumenten. Neben einem Nutzermodell für die Relevanzbewertung von Suchergebnissen in akademischen Informationssystemen präsentiert sie einen Vorschlag für eine explizite Unterscheidung zwischen Relevanzmerkmalen, Relevanzkriterien und Relevanzfaktoren. Damit trägt der Artikel auch zur terminologischen Präzisierung in der informationswissenschaftlichen Forschung bei.

Das Projekt AAPVL hat sich mit dem Lebensmittelhandel im Internet beschäftigt und ein prototypisches System entwickelt, wie die Lebensmittelkontrolle auf dem Online-Markt teilweise automatisiert werden kann. Sebastian
Sünkler, Alexandra Krewinkel, Mareike Gleissner, Dorle Osterode, Boris Tolg, Martin Holle und Dirk Lewandowski berichten in ihrem Aufsatz „Entwicklung und Anwendung einer Software zur automatisierten Kontrolle des Lebensmittelmarktes im Internet unter Verwendung informationswissenschaftlicher Methoden" von den Ergebnissen des Projektes. Sie zeigen insbesondere auch, wie spezifisch informationswissenschaftliche Fragestellungen und Kompetenzen in einem auf den ersten Blick vielleicht wenig naheliegenden Anwendungsbereich umgesetzt werden.

Der Beitrag „Das Relevance Assessment Tool - Eine modulare Software zur Unterstützung bei der Durchführung vielfältiger Studien mit Suchmaschinen“ von Sebastian Sünkler und Dirk Lewandowski stellt eine in der Forschungsgruppe Search Studies entwickelte Forschungssoftware vor. Damit können auf Basis von vorgegebenen Suchanfragen kommerzielle Suchmaschinen wie Google und Bing automatisch abgefragt und die Ergebnisse dann Juroren zur Bewertung vorgelegt werden. Die Software unterstützt die Durchführung einer Vielzahl unterschiedlich konzipierter Suchmaschinenstudien. Wie das Tool konkret eingesetzt werden kann, wird im Beitrag an einer Auswahl bereits durchgeführter Projekte gezeigt.

Wir wünschen Ihnen eine erkenntnisreiche und anregende Lektüre!

Dirk Lewandowski und Ulrike Spree 